\title{
On the Capacity of CPM MIMO Systems over Band-Limited Channels
}

\author{
Guowei Lei ${ }^{1,2}$, Xuefang Xiao ${ }^{3}$ \\ ${ }^{1}$ School of Science, Jimei University, Xiamen, China \\ ${ }^{2}$ School of Electronic engineering, Beijing University of Posts and Telecommunications, Beijing, China \\ ${ }^{3}$ School of Optoelectronics and Communication Engineering, Xiamen University of Technology, Xiamen, China \\ Email: gwlei@163.com
}

How to cite this paper: Lei, G.W. and Xiao, X.F. (2017) On the Capacity of CPM MIMO Systems over Band-Limited Channels. Int. J. Communications, Network and System Sciences, 10, 69-75. https://doi.org/10.4236/ijcns.2017.108B008

Received: May 7, 2017

Accepted: August 11, 2017

Published: August 14, 2017

\begin{abstract}
Capacity analysis is a fundamental and important issue for continuous phase modulation (CPM) signals. In the letter, we investigate the capacity formula of CPM MIMO systems. Using Finite State Machine (FSM), the CPM symbols can be modeled as Markov source by combining channel and CPM modulation. Thus the capacity of CPM signals can be derived in form of the erroneous probability and normalized CPM bandwidth. In addition, the capacity of CPM MIMO systems is derived over Gaussian channels and Rayleigh channels. Finally, numerical simulations are implemented according to various parameters such as modulation scheme, modulation index $h$, memory length $L$, and antenna configuration.
\end{abstract}

\section{Keywords}

Capacity, Continuous Phase Modulation, MIMO, Rayleigh Channel

\section{Introduction}

CPM is a non-linear modulation with significant advantages for low power, low cost and spectrum efficiencies. Notwithstanding these favorable features, however, it is difficult to compute the capacity of a channel using CPM due to several factors such as the continuous phase and the nonlinearity of modulation [1]. In [1], the CPM in AWGN channels was considered equivalent to a multiple access channel using Laurent decomposition, which was an attempt to solve theoretical problem for Shannon capacity of CPM. In addition to this, symmetric information rate (SIR) is an alternative measure for Shannon capacity. It can achieve a lower bound of capacity constrained on the modulation format [2]. By modeling CPM using FSM (Finite State Machine), SIR can be estimated via BCJR algorithm [3]. Hereafter, a reliable estimate of the capacity was extended to genera- 
lized CPM scheme [4]. On the other hand, the theoretical limits on the bandwidth efficiency of CPM signals were estimated by implementing the Carson's Rule bandwidth measure [5].

Unfortunately, there is not closed-form formula so far even for point-to-point link. Let alone the analysis of CPM in MIMO environment. In this letter, we derive the upper bounds of capacity for CPM systems over Gaussian and Rayleigh band-limited channels. First of all, the symmetry information rate (SIR) is given in form of mutual information between input and output. Then SIR is calculated in terms of Markov source for M-ary CPM signals [6]. Further, the probability of an erroneous decision over Gaussian and Rayleigh channels is obtained respectively. Finally, the approximate upper-bounded capacity over band-limited channels can be derived for CPM MIMO systems.

\section{Signal Model and Capacity Formulation}

Capacity portrays the achievable rate at which information can be reliably transmitted over a communications channel. Unlike linear modulations such as PSK and QAM, the spectral properties of CPM signals generally depend on the complete statistical description.

\subsection{Signal Model}

The baseband CPM signal transmitted by source has complex form as [7].

$$
x(t ; \vec{D})=\sqrt{E_{s}} \exp \left[j\left(\varphi(t ; \vec{D})+\theta_{0}\right)\right]
$$

and

$$
\varphi(t ; \vec{D})=2 \pi h \sum_{-\infty}^{+\infty} D_{n} q(t-n T)
$$

where $E_{s}$ is the power of CPM signal, $T$ is the symbol period, $h$ is the modulation index, $j=\sqrt{-1},\left\{D_{n}\right\}$ is the sequence of independent information symbols drawn from the alphabet $\{ \pm 1, \pm 3, \ldots\}, \theta_{0}$ is initial phase.

The function $q(t)$ in (2) is defined as the phase smoothing response of the CPM signals.

$$
q(t)=\int_{0}^{t} g(t) d t
$$

The shape of $g(t)$ in (3) defines a family of CPM schemes, where two widely used types such as rectangular pulse with pulse length $L$ ( $L R E C)$ and raised cosine pulse with pulse length $L(L R C)$ are given in Table 1 .

\subsection{Capacity Formulation}

The block diagram of a CPM modulation channel is illustrated in Figure 1. Input symbols that are drawn from $\{0,1, \cdots, M\}$ are mapped into $D_{i}(k) \in\{-(M-$ $1), \cdots,(M-1)\}$. Herein the channel combined with CPM modulation model would be better identified as a discrete memory-less channel (DMC). As the inputs of CPM modulator are independent and uniformly distributed random variables, a generalized CPM scheme may be modeled using Finite State Machine 
Table 1. Pulse-shaping function of CPM schemes.

\begin{tabular}{cc}
\hline Modulation Scheme & Pulse-Shaping Function $g(t)$ \\
\hline LRC & $\frac{1}{2 L T}\left[1-\cos \left(\frac{2 \pi t}{L T}\right)\right], 0 \leq t \leq L T$ \\
LREC & $\frac{1}{2 L T}, 0 \leq t \leq L T$ \\
\hline
\end{tabular}

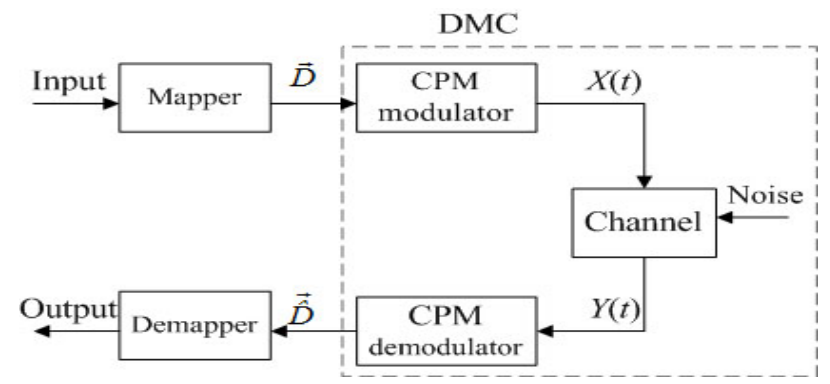

Figure 1. Block diagram of a CPM modulation channel.

(FSM).

Thus, the SIR (bit/channel use) may be calculated in terms of Markov source for M-ary CPM signals (as shown in Figure 1). In Gaussian channels, received signal is represented as

$$
y=x+w
$$

where the noise $w$ has zero-mean and variance $N_{0}$. The mutual information between the input signal $X$ and the output signal $Y$ is therefore obtained as the length $N$ goes to infinity, i.e.,

$$
S I R=\mathrm{I}(\boldsymbol{D} ; \hat{\boldsymbol{D}})=\lim _{N \rightarrow \infty} \frac{1}{N} \mathrm{I}(\mathrm{X}(t, \vec{D}(N-1)) ; \mathrm{Y}(t, \overrightarrow{\hat{D}}(N-1)))
$$

Invoking information theory, the mutual information can be written as

$$
\mathrm{I}(\boldsymbol{D} ; \hat{\boldsymbol{D}})=\mathrm{H}(\hat{\boldsymbol{D}})-\mathrm{H}(\hat{\boldsymbol{D}} \mid \boldsymbol{D})
$$

To calculate each item in (6), we have

$$
\begin{aligned}
\mathrm{H}(\hat{\boldsymbol{D}}) & =\sum_{j=1}^{M} p\left(\hat{D}_{j}\right) \log p\left(\hat{D}_{j}\right) \\
& =\sum_{j=1}^{M} \sum_{i=1}^{M} p\left(\hat{D}_{j} \mid D_{i}\right) p\left(D_{i}\right) \log \left[\sum_{i=1}^{M} p\left(\hat{D}_{j} \mid D_{i}\right) p\left(D_{i}\right)\right] \\
\mathrm{H}(\hat{\boldsymbol{D}} \mid \boldsymbol{D}) & =\sum_{j=1}^{M} \sum_{i=1, i \neq j}^{M} p\left(\hat{D}_{j} \mid D_{i}\right) p\left(D_{i}\right) \log p\left(\hat{D}_{j} \mid D_{i}\right) \\
& =\sum_{j=1}^{M} \sum_{i=1, i \neq j}^{M} p_{e} p\left(D_{i}\right) \log p_{e}
\end{aligned}
$$

To compute (7) and (8), the probability of an erroneous decision over Gaussian channels is written as [7]

$$
p_{e}^{\text {Gauss }}=\mathrm{Q}\left(\left(d_{\min }^{2} \cdot \gamma_{\text {Gauss }}\right)^{1 / 2}\right)
$$

where $\gamma_{\text {Gauss }}=E_{b} / N_{0}$, and $d_{\min }^{2}$ denotes the minimal distance with respect to the 
pair of sequences $D_{n}$ and $\hat{D}_{n}$. In practical calculation, $d_{\min }^{2}$ should be of the form [7]

$$
\begin{aligned}
d_{\min }^{2} & =\lim _{N \rightarrow \infty} d_{\min , N}^{2} \\
& =\lim _{N \rightarrow \infty} 2 E_{s} \cdot \min _{\bar{D}}\left\{N-\frac{1}{N} \int_{0}^{N T} \cos [\varphi(t, \vec{D})] d t\right\}
\end{aligned}
$$

In Rayleigh fading channels, the CPM-MIMO system is comprised of $N_{T}$ transmit antenna and $N_{R}$ receive antenna. The received signal vectors are given by

$$
\boldsymbol{Y}=\boldsymbol{H} \boldsymbol{X}+\boldsymbol{W}
$$

where $\boldsymbol{X}$ are CPM signal vectors that are transmitted from $N_{T}$ antennas, $H$ is the channel matrix, in which each entry is independent and identical distributed (i.i.d) Rayleigh fading.

For the orthogonal design with $N_{T} \times N_{R}$ antennas, the received SNR can be given by expression in [8] as

$$
\begin{aligned}
\gamma_{\text {Rayleigh }} & =\sum_{n_{t}=1}^{N_{T}} \sum_{n_{r}=1}^{N_{R}}\left|h_{n_{t} n_{r}}\right|^{2} \frac{E_{b}}{N_{T} N_{0}} \\
& =\sum_{n_{t}=1}^{N_{T}} \sum_{n_{r}=1}^{N_{R}} \rho_{n_{t} n_{r}} \frac{E_{b}}{N_{T} N_{0}}
\end{aligned}
$$

As the magnitude of $h_{n t n r}$, i.e. $\left|h_{n t n n}\right|$, is Rayleigh distributed, $\rho=\left|h_{n t n r}\right|^{2}$ is exponentially distributed. Hence, the probability of an erroneous decision over Rayleigh channels can be calculated to

$$
p_{e}^{\text {Rayleigh }}=\int_{\rho} \mathrm{Q}\left(\left(d_{\min }^{2} \cdot \gamma_{\text {Rayleigh }}(\rho)\right)^{1 / 2}\right) p(\rho) \mathrm{d} \rho
$$

Since $\mathrm{Q}(\cdot)$ function can be simplified as $\mathrm{Q}(r) \leq(1 / 2) \exp \left(-r^{2} / 2\right)$, (13) can be approximately upper-bounded by

$$
p_{e}^{\text {Rayleigh }} \leq \frac{1}{2}\left(1+\frac{d_{\min }^{2} E_{b}}{2 N_{T} N_{0}}\right)^{-N_{T} N_{R}}
$$

On the other hand, the normalized CPM bandwidth is confined according to the parameters of CPM signals [5].

As for LREC scheme, the Carson's Rule bandwidth of the CPM signal is given by

$$
B \geq \frac{h}{T} \sqrt{\frac{M^{2}-1}{3 L}}+\frac{1}{L T}
$$

As for LRC scheme, the Carson's Rule bandwidth of the CPM signal is given by

$$
B \geq \frac{h}{T} \sqrt{1.5 \frac{M^{2}-1}{3 L}}+\frac{1.5}{L T}
$$

Finally, the CPM capacity can be given by

$$
\mathrm{R} \leq \mathrm{C}=\frac{\mathrm{I}(\boldsymbol{D} ; \hat{\boldsymbol{D}})}{B T} \leq \frac{\mathrm{H}(\hat{\boldsymbol{D}})-\mathrm{H}(\hat{\boldsymbol{D}} \mid \boldsymbol{D})}{h \sqrt{\alpha \frac{M^{2}-1}{3 L}+\frac{\alpha}{L}}} \quad \begin{cases}\text { LREC } & \alpha=1 \\ \text { LRC } & \alpha=1.5\end{cases}
$$




\section{Numerical Results and Discussions}

In this section, we provide simulated results to analyze the channel capacity of CPM MIMO system. Figure 2 depicts the capacity of CPM with $M=2, L=1$ REC over Gaussian channels. In high signal to noise ratio (SNR), the capacity (bits/s/Hz) turns to be larger as modulation index $h$ is decreased. This may attribute to the constraint of bandwidth. Whereas in low SNR, the capacity for $h=0.4,0.5$ and 0.6 appears close to each other.

As a comparison, the plots of channel capacity are given regardless of bandwidth. It is observed in Figure 3 that, the SIR will become larger as modulation index $h$ is increased. It is demonstrated that the minimal distance $d_{\min }^{2}$ should play an important role in the erroneous probability.

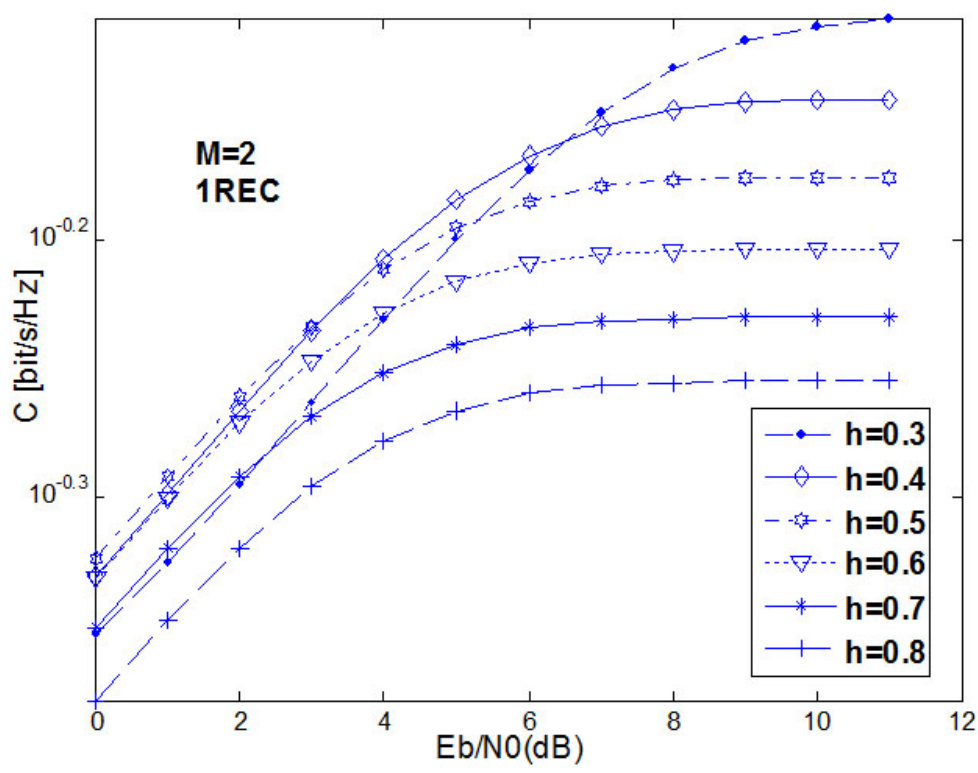

Figure 2. Capacity of CPM with $M=2,1 \mathrm{REC}$ over Gaussian channels.

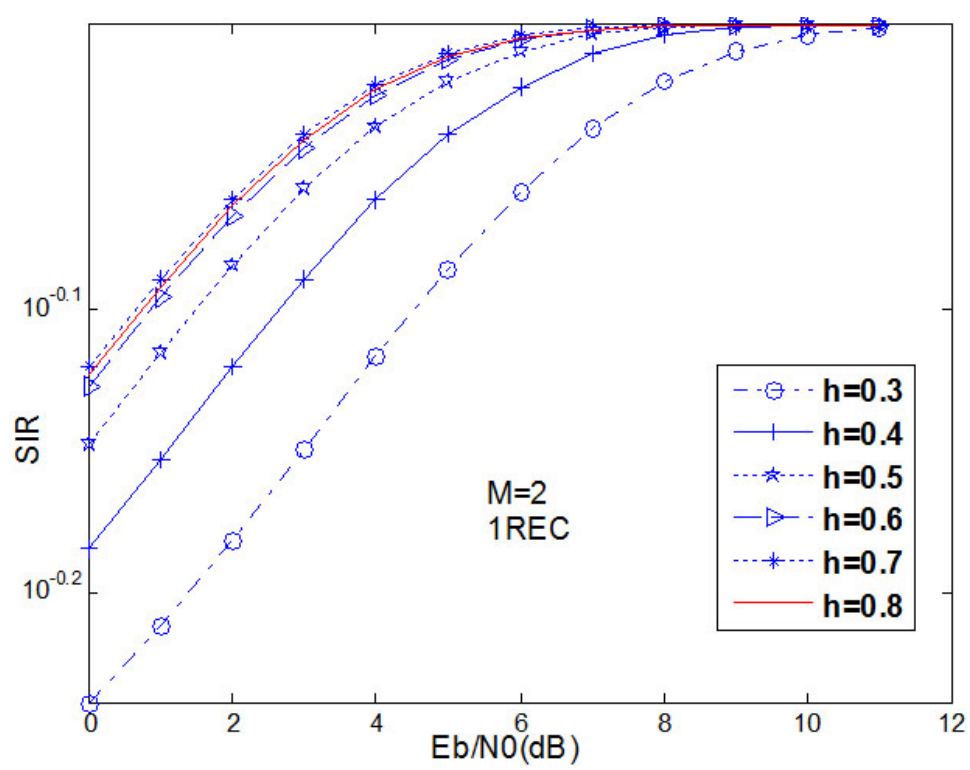

Figure 3. SIR of CPM with $M=2,1$ REC over Gaussian channels. 
The capacity of CPM with $M=2, h=0.5$ for REC and RC ( $L=1$ and 2$)$ over Gaussian channels are shown in Figure 4. Although the minimal distance $d_{\text {min }}^{2}$ for LREC and LRC with $M=2, h=0.5$ is same, the capacity of CPM for LREC is larger than that for LRC. It is owing to the fact that the normalized CPM bandwidth for LREC is narrower than that for LRC. Similarly, the capacity of CPM for $L=2$ REC should be larger than that for $L=1$ REC.

Then, our next concern is about CPM MIMO systems with $N_{T} \times N_{R}$ antennas over Rayleigh channels. Under the condition that $\mathrm{M}$ is same (as plotted in Figure 5), the capacity of $\left(N_{t}, N_{r}\right)=(2,2)$ should be the largest due to its diversity gain. On the other hand, when the configuration $\left(N_{t}, N_{r}\right)$ of CPM MIMO systems is same, the capacity for $M=4$ is entirely larger than that for $M=2$.

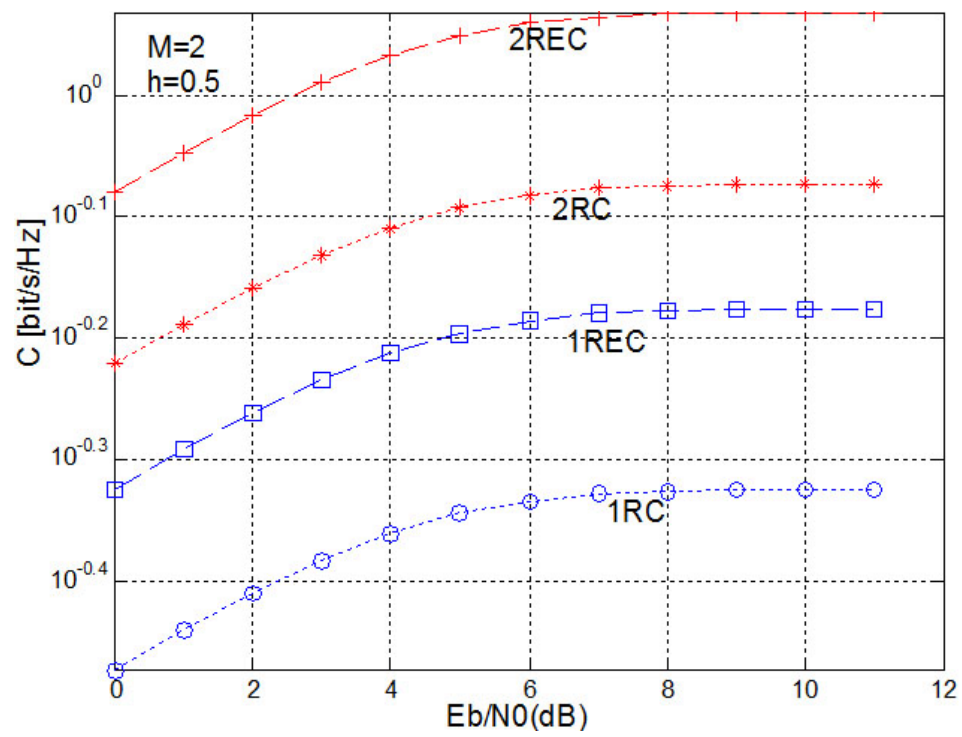

Figure 4. Capacity of CPM with $M=2, h=0.5$ for REC and RC ( $L=1$ and 2) over Gaussian channels.

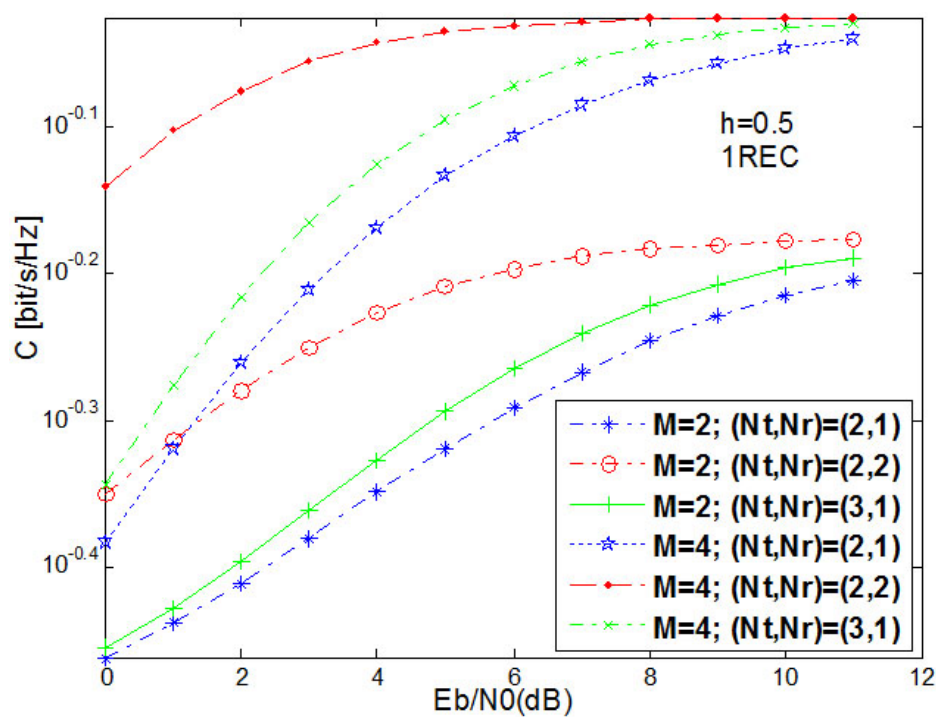

Figure 5. Capacity of CPM with $h=0.5,1$ REC for $M=2$ and 4 over Rayleigh channels. 


\section{Conclusion}

In this paper, we investigate the capacity of CPM MIMO systems over band-limited channels. We give a formulation of CPM capacity in form of SIR and normalized CPM bandwidth. For this purpose, the erroneous probability of CPM MIMO systems over Gaussian channels and Rayleigh channels is given and derived respectively. Finally, the capacity of CPM MIMO systems is simulated and evaluated according to various parameters such as modulation scheme, modulation index h, memory length $\mathrm{L}$, and antenna configuration.

\section{Acknowledgements}

This work is supported by Foundation project of department of education of Fujian Province (JAT160260), Li Shangda Discipline Construction Fund of Jimei University, Pre-research project of National Natural Science Foundation of China (XYK201406).

\section{References}

[1] Goldsmith, A.J. and Yu, K. (2002) Linear Models and Capacity Bounds for Continuous Phase Modulation. IEEE International Conference on communications (ICC 2002), New York, April 28-2 May 2002, 722-726.

[2] Zhang, X.X. and Fitz, M.P. (2003) Symmetric Information Rate for Continuous Phase Channel and BLAST Architecture with CPM MIMO System. IEEE International Conference on communications (ICC 2003), Alaska, 11-15 May 2003, 30513055.

[3] Bahl, L.R., Cocke, J., Jelinek, F., et al. (1974) Optimal Decoding of Linear Codes for Minimizing Symbol Error Rate. IEEE Transactions on Information Theory, 20, 284287. https://doi.org/10.1109/TIT.1974.1055186

[4] Padmanabhan, K., Ranganathan, S. and Sundaravaradhan, S.P. (2005) General CPM and its Capacity. International Symposium on Information Theory (ISIT 2005), Adelaide, 4-9 September 2005, 750-754. https://doi.org/10.1109/ISIT.2005.1523437

[5] Kuo, C.H. and Chugg, K.M. (2005) Improving the Bandwidth Efficiency and of CPM Signals via Shaping and Iterative Detection. IEEE Military Communications Conference (MILCOM 2005), Atlantic, 17-20 October 2005, 596-602.

[6] Barbieri, A., Cero, A., Piemontese, A., et al. (2007) Markov Capacity of Continuous Phase Modulation. International Symposium on Information Theory (ISIT 2007), Nice, 24-29 June 2007, 161-165. https://doi.org/10.1109/ISIT.2007.4557220

[7] Aulin, T. and Sundberg, C.-E.W. (1981) Continuous Phase Modulation-Part I: Full Response Signaling. IEEE Transactions on Communications, 29, 196-209. https://doi.org/10.1109/TCOM.1981.1095001

[8] Lei, G.W., Liu, Y.A. and Xiao, X.F. (2016) Effect of Imperfect CSI on STBC-MISO System via Antenna Selection. IET Signal Processing, 10, 115-124. https://doi.org/10.1049/iet-spr.2015.0127 
Submit or recommend next manuscript to SCIRP and we will provide best service for you:

Accepting pre-submission inquiries through Email, Facebook, LinkedIn, Twitter, etc. A wide selection of journals (inclusive of 9 subjects, more than 200 journals)

Providing 24-hour high-quality service

User-friendly online submission system

Fair and swift peer-review system

Efficient typesetting and proofreading procedure

Display of the result of downloads and visits, as well as the number of cited articles Maximum dissemination of your research work

Submit your manuscript at: http://papersubmission.scirp.org/

Or contact ijcns@scirp.org 